\title{
Exploring the Effects of Different Cross-Linkers on Lignin-Based Thermoset Properties and Morphologies
}

\author{
Iuliana Ribca, Marcus E. Jawerth, Calvin J. Brett, Martin Lawoko, Matthias Schwartzkopf, \\ Andrei Chumakov, Stephan V. Roth, and Mats Johansson*
}

Cite This: https://dx.doi.org/10.1021/acssuschemeng.0c07580

Read Online

ABSTRACT: The search for sustainable material solutions has put lignin as one of the prime candidates for aromatic building blocks in macromolecular materials. The present study aimed to demonstrate how lignin-based thermoset resins can be utilized in combination with different cross-linkers. Kraft lignin was used to produce thermosets with tunable mechanical and morphological properties. The lignin-based thermosets were obtained via a thermally induced thiol-ene reaction. The first part of this work was focused on Kraft lignin solvent fractionation and chemical modification of the ethanol soluble fraction. Chemical analysis indicated that the allylation process was selective toward phenolic hydroxyl groups. SAXS and SEM studies demonstrated that solvent fractionation and allylation processes affected the molecular and
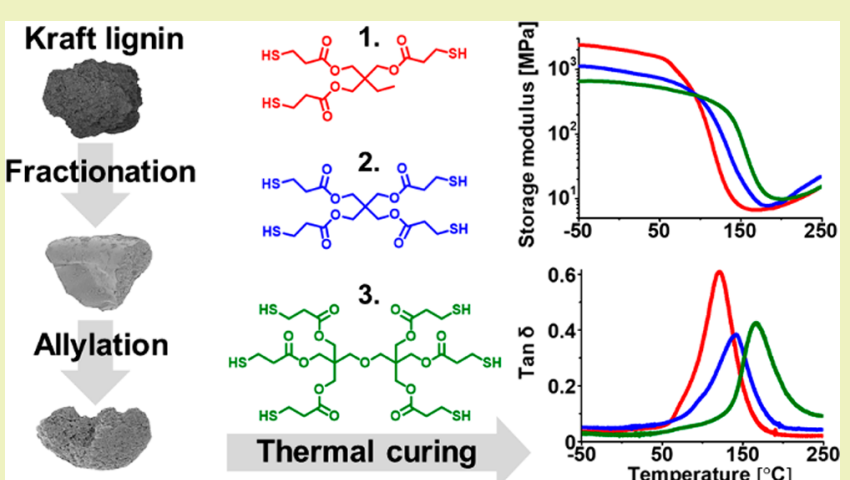
nanoscale morphological characteristics of lignin. The second part's focus was on how the properties of thermosets can be tuned by using three different cross-linkers. The dynamic mechanical and morphological properties of three different thermosets were investigated via DMA, SAXS, and WAXS techniques. The three different thermosets exhibit similar molecular morphology but different storage modulus and glass transition temperature. In this work, it was shown that despite lignin's heterogeneity it was possible to produce thermosetting materials with tunable properties.

KEYWORDS: Kraft lignin, Solvent fractionation, Selective allylation, Thiol-ene thermoset, Mechanical properties, Small- and wide-angle X-ray scattering

\section{INTRODUCTION}

A thermosetting material is formed by polymerization and cross-linking reactions between polyfunctional monomers leading to a three-dimensional network. The most used monomers are derived from oil-based resources. The drive toward sustainable development builds the way to a global tendency of replacing fossil-based polymers with ones from renewable resources. Aromatic compounds are widely used as building blocks for thermosetting materials since they confer higher thermal stability, stiffness, and glass transition temperature $\left(T_{g}\right)$. The epoxy resins represent $70 \%$ of the total market of polymeric resins. ${ }^{1}$ One of the most used aromatic monomers for synthesis of epoxy resins is derived from bis(4-hydroxyphenylene)-2, 2-propane (bisphenol A, BPA). This compound has a negative impact on human health and the environment. ${ }^{2}$ Due to its relatively high aromaticity, lignin has the potential to be an excellent alternative to the BPAbased thermosets.

Lignin is one of the main wood components that acts as natural glue, binding together the cellulose fibers. This aromatic, complex, and heterogeneous polymer is derived from the oxidative coupling of coniferyl, sinapyl, and p- coumaryl alcohol. ${ }^{3}$ Lignin is available in high quantities as a byproduct from pulp and paper industries. ${ }^{4}$ During the pulping process, its chemical structure undergoes significant changes by fragmentation and condensation. ${ }^{5}$ It has been suggested that lignin fragmentation into lower molecular weight compounds results into formation of new phenolic $\mathrm{OH}$ groups. ${ }^{6}$

The technical lignins obtained by different processes, such as Kraft, soda, or organosolv, vary in molecular weight distribution, polydispersity, types of functional groups (mainly phenolic and aliphatic hydroxyl groups, and carboxylic acid groups), and interunit linkages. ${ }^{7}$ The heterogeneity and poor solubility of lignin limit its application in high value-added materials. To resolve this issue, technical lignin can be fractionated and chemically modified by various approaches. In order to control the molecular weight distribution and

Received: October 15, 2020

Revised: December 8, 2020 
polydispersity, various fractionation methodologies, such as membrane filtration, sequential precipitation, solvent screening, or microwave processing, can be applied. ${ }^{8-12}$ Another approach is to degrade the technical lignin into monomeric units with subsequent purification and retrieval, for example, vanillin and other derivatives. ${ }^{13,14}$ Utilization of lignin as an aromatic source for polymers can thus be achieved in several different ways. Extensive work has, for example, been done on modifying vanillin for polymer use. ${ }^{15}$ Utilizing lignin fractions instead of monomeric units has the advantage of retrieving the raw material more efficiently. The main challenge, however, is to obtain reproducible and well-characterized fractions.

Lignin incorporation in functional materials requests not only good solubility but also thermal stability and reactive sites. Oxypropylation, allylation, epoxidation, acetylation, and silylation are just a few pathways for technical lignin modification found in the literature. ${ }^{16-21}$ The lignin allylation approach developed by Jawerth et al. represents a promising route for further utilization of lignin as a thermoset constituent. $^{21}$ It was clearly shown that the final thermoset's properties could be tailored by using different lignin fractions. $^{22}$

The free radical thiol-ene reaction, discovered in 1926, has drawn increased interest during the last decades, especially for thermoset polymers, due to the versatility in varying the alkene monomers. ${ }^{23-28}$ This efficient reaction allows the use of otherwise difficult to homopolymerize alkene monomers such as di- or trisubstituted alkenes. ${ }^{29}$ Thiol-ene polymerization also exhibits advantages, such as lower sensitivity to oxygen inhibition and a stepwise reaction. A drawback, however, is the often rather low $T_{g}$ and modulus caused by the formed flexible thiol-ether bond. ${ }^{2}$

In this study, a technical lignin fraction is retrieved and chemically functionalized to form thermosets using three different cross-linking agents. It is elucidated how the final thermoset properties can be tailored by the choice of different polyfunctional thiol cross-linkers to cure the lignin-based resins. Furthermore, the study includes structural characterization using wide- and small-angle X-ray scattering (WAXS, SAXS) to reveal details of the morphology of the final thermoset and to yield information on the structure-property relationship.

\section{EXPERIMENTAL SECTION}

Materials. Softwood LignoBoost Kraft lignin (KL) was supplied by Stora Enso (Finland). Ethyl acetate (EtOAc, $\geq 99 \%$ ), ethanol (EtOH, $\geq 99.8 \%)$, methanol $(\mathrm{MeOH}, \geq 99.8 \%)$, acetone ( $\geq 99.5 \%)$, and hydrochloric acid $(\mathrm{HCl}, 37 \%)$ were purchased from VWR International. Sodium hydroxide $(\mathrm{NaOH}, \geq 98 \%)$, allyl chloride (98\%), and trimethylolpropane tris(3-mercaptopropionate) (3TMP, $\geq 95 \%$ ) were obtained from Sigma-Aldrich. Pentaerythritol tetrakis(3mercaptopropionate) (4PER, 95\%) was supplied by Bruno Bock Chemische Fabrik GmbH \& Co., and dipentaerythritol hexakis-(3mercaptopropionate) (6DPER, $>93 \%$ ) was purchased from Tokyo Chemical Industry (TCI). Silicon molds were prepared with a silastic $\mathrm{T}-2$ base/curing agent $(10: 1 \mathrm{w} / \mathrm{w})$, which were obtained from Dow Corning. All other chemicals were of analytical grade and used as received from Sigma-Aldrich.

Procedures. Purification and Solvent Fractionation of KL. The $\mathrm{KL}$ was washed to reduce ash and impurities. Roughly $20 \mathrm{~g}$ of lignin were immersed in $400 \mathrm{~mL}$ of deionized water and magnetically stirred at $60{ }^{\circ} \mathrm{C}$ for $2 \mathrm{~h}$. Lignin was separated from the washing medium by filtration, and this procedure was repeated until the $\mathrm{pH}$ of the aqueous permeate was above 5.5. The residue was dried in a vacuum oven at
$50{ }^{\circ} \mathrm{C}$ for $20 \mathrm{~h}$ to ensure a high dry content, which is assumed to be approximately $95 \%$ after this step.

The washed and dried lignin (KL Washed) was then sequentially solvent-fractionated using $\mathrm{EtOAc}, \mathrm{EtOH}, \mathrm{MeOH}$, and acetone following the previously developed protocol by Duval et al. with minor modifications. ${ }^{9}$ Roughly $(15 \pm 5) \mathrm{g}$ of $\mathrm{KL}$ were dissolved in $150 \mathrm{~mL}$ of EtOAc (solvent volume was adjusted in order to maintain lignin concentration of $100 \mathrm{~g} / \mathrm{L}$ ) and stirred using a magnetic stirrer at room temperature for $2.5 \mathrm{~h}$. The solution was filtered through filter paper (Grade 3 Munktell, Sweden), and the insoluble fraction was further washed with EtOAc. To recover the separated lignin, the solvent was removed by a rotary evaporator $\left(\leq 50^{\circ} \mathrm{C}\right)$, and the sticky extract was dried overnight at $50{ }^{\circ} \mathrm{C}$ in a vacuum oven. The dried lignin was redissolved in $10-15 \mathrm{~mL}$ of acetone and precipitated in deionized water to give a concentration of $\approx 7,19$, and $40 \mathrm{mg} / \mathrm{mL}$, respectively. The dispersion was freeze-dried to obtain a fine powder of the EtOAc soluble lignin fraction. The insoluble residue from the filtration was immersed in the next solvent, and the same procedure was repeated with the remaining solvents. Thus, four different soluble lignin fractions were obtained in function of the used solvent: $\mathrm{KL}$ EtOAc, $\mathrm{KL} \mathrm{EtOH}, \mathrm{KL} \mathrm{MeOH}, \mathrm{KL}$ Acetone, and one insoluble $\mathrm{KL}$ Insoluble. All fractions were dried at $50^{\circ} \mathrm{C}$ in a vacuum oven for $20 \overline{\mathrm{h}}$ before further use.

Selective Allylation of $K L_{-} E t O H$. Allylated lignin was synthesized according to a previously reported method by Jawerth and coworkers. ${ }^{21,22}$ Only the KL_EtOH was selectively allylated toward the phenolic hydroxyl functionalities with an excess of $\mathrm{NaOH}$ and allyl chloride (approximately $1.4 \mathrm{mmol}$ per $1 \mathrm{mmol}$ of phenolic hydroxyl functionalities). Here $1 \mathrm{~g}$ of $\mathrm{KL} \_$EtOH $(4.6 \mathrm{mmol}$ of phenolic hydroxyl groups) was added to a two-necked round-bottomed flask containing $55 \mathrm{~mL}$ of an $\mathrm{EtOH} / \mathrm{NaOH}(60: 40, \mathrm{v} / \mathrm{v})$ solution. The flask was equipped with a condenser and immersed in an oil bath. The second neck of the flask was sealed with a rubber septum. The mixture was magnetically stirred at $65{ }^{\circ} \mathrm{C}$ until a homogeneous dark-brown solution was obtained. Allyl chloride was then added dropwise using a syringe through the septum, and the reaction media was vigorously stirred for $40 \mathrm{~h}$. Subsequently, the mixture was let to cool to ambient temperature, and $50 \mathrm{~mL}$ of deionized water was added to dilute the system. The allylated lignin was precipitated by adding $0.1 \mathrm{M} \mathrm{HCl}$ dropwise and filtered by a Büchner funnel with filter paper (Grade 3 Munktell, Sweden). The obtained precipitate was washed with deionized water to remove traces of acid and salt. Finally, the obtained product was redissolved in $15 \mathrm{~mL}$ of acetone, precipitated in $200 \mathrm{~mL}$ of deionized water, and freeze-dried. The product was recovered as a light-brown fine powder (A-KL_EtOH) and was dried in a vacuum oven at $50{ }^{\circ} \mathrm{C}$ for $20 \mathrm{~h}$ before further utilization. An overview of the $\mathrm{KL} \_$EtOH allylation can be seen in Scheme 1 .

Scheme 1. Selective Allylation Towards Phenolic OH of KL_EtOH

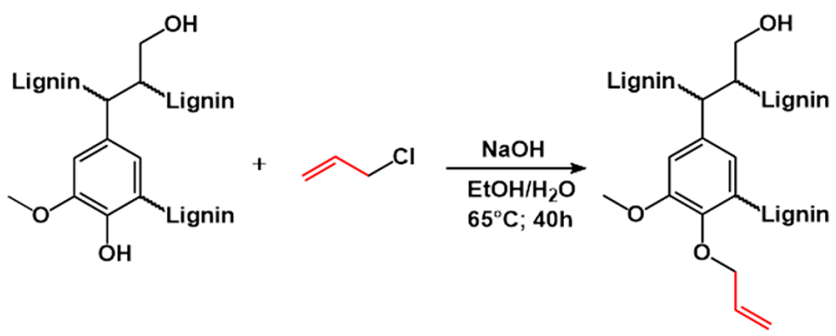

Preparation and Curing of Thiol-Ene Thermosetting Resins. Lignin-based thermosets were prepared based on the procedure previously described by Jawerth et al. ${ }^{21}$ In this work, trimethylolpropane tris(3-mercaptopropionate), pentaerythritol tetrakis(3-mercaptopropionate), and dipentaerythritol hexakis-(3-mercaptopropionate), three thiol cross-linkers, were used to prepare thermosets with similar allylated lignin content. The specific cross-linking agents contain relatively the same number of millimoles of functional groups per 
gram of cross-linking agent even though they have different numbers of functional groups per molecule (Table 1). The molar ratio between

Table 1. Molecular Weight of Crosslinkers and Distribution of Functional Groups

\begin{tabular}{cccc}
$\begin{array}{c}\text { Thiol } \\
\text { cross-linkers }\end{array}$ & $\begin{array}{c}\text { Molecular weight } \\
(\mathrm{g} / \mathrm{mol})\end{array}$ & $\begin{array}{c}\text { No. of SH } \\
\text { groups/molecule }\end{array}$ & $\begin{array}{c}\text { mmol SH/g } \\
\text { cross-linker }\end{array}$ \\
3TMP & 399 & 3 & 7.5 \\
4PER & 489 & 4 & 8.2 \\
6DPER & 783 & 6 & 7.6 \\
\hline
\end{tabular}

the thiol and ene functional groups were kept at 1:1. Thermosets resins were prepared by mixing approximately $50 \mathrm{mg}$ of A-KL_EtOH with $25 \mathrm{mg}$ of either the tri-, tetra-, or hexafunctional thiol crosslinkers (Table 1). To ensure a thorough mixing of the lignin resin with cross-linkers, $150 \mathrm{mg}$ of EtOAc was used as a cosolvent. The resins were cured in soft silicon molds so that the final samples could be retrieved without breaking due to their brittle nature. To avoid bubble formation, the silicon molds were covered with glass Petri dishes to slow the evaporation rate during the solvent casting. The lignin-based resins were left in the fume hood for $24 \mathrm{~h}$. Afterward, the homogeneous samples were transferred into an oven and cured at 125 ${ }^{\circ} \mathrm{C}$ for $20 \mathrm{~h}$. The obtained thermosets were taken out of the oven, let to cool to room temperature, and carefully removed from the silicon molds. The three different resins/thermosets $(\mathrm{R} / \mathrm{T})$ were denoted according to the specific thiol cross-linkers used as follow: $\mathrm{R} / \mathrm{T}$ KL_EtOH_3TMP, R/T-KL_EtOH_4PER, and R/T-KL_EtOH 6 DPER.

Characterization Techniques. Fourier Transform Infrared Spectroscopy (FT-IR). A PerkinElmer Spectrum 100 instrument was used to determine the chemical composition of modified lignin specimens and lignin-based thermosets. FT-IR spectra were acquired in attenuated total reflection (ATR) mode with an MKII Golden Gate accessory (Specac Ltd.) equipped with a diamond crystal. Each measurement was averaged over 16 scans between 4000 and $600 \mathrm{~cm}^{-1}$ with a resolution of $4 \mathrm{~cm}^{-1}$ at room temperature. All spectra were baseline corrected and normalized to the $1732 \mathrm{~cm}^{-1}$ carbonyl signal via PerkinElmer Spectrum software V10.5.1.

Ash Content. The ash content was determined by a muffle furnace (M9-1200) according to the T 211 om-02 method. Each sample, with a weight of $0.50 \pm 0.01 \mathrm{~g}$, was analyzed three times, and the average values were reported.

Inductively Coupled Plasma-Optical Emission Spectroscopy (ICP-OES). The determination of inorganics in $\mathrm{KL}$ and $\mathrm{KL}$-Washed was done via a Thermo Scientific iCAP 6000 series instrument. The sample preparation and digestion were done following the US EPA SW-846 method 3050A with some modifications. The dried lignin sample $(0.50 \pm 0.01 \mathrm{~g})$ was added into a beaker and covered with a watch glass. Then, $5 \mathrm{~mL}$ of concentrated nitric acid $\left(\mathrm{HNO}_{3}, 65 \%\right)$ was added, and the sample was heated for $15 \mathrm{~min}$ to a gentle reflux. Afterward, the sample was allowed to cool, and $2.5 \mathrm{~mL}$ of $\mathrm{HNO}_{3}$ was added. The sample was heated for another $30 \mathrm{~min}$. For complete oxidation, the last steps were repeated one more time. Here, $1.5 \mathrm{~mL}$ of $30 \%$ of hydrogen peroxide solution $\left(\mathrm{H}_{2} \mathrm{O}_{2}\right)$ was added into the cooled beaker. The mixture was heated until the effervescence subsided and then was allowed to cool. This step was repeated one more time but with only $1 \mathrm{~mL}$ of $\mathrm{H}_{2} \mathrm{O}_{2}$. The cooled sample was diluted to a $50 \mathrm{~mL}$ volume with Milli- $Q$ water. Before the analysis, the solution was filtered using $0.2 \mu \mathrm{m}$ filters. Standard solutions were used to obtain the calibration curve. Elements' concentrations were determined at different wavelengths, and the average values were taken for the data evaluation.

Size Exclusion Chromatography (SEC). Molecular weight distribution and polydispersity $(\boxplus)$ of lignin samples were determined using a SECcurity 1260 infinity GPC System (Polymer Standards Service, Germany). The instrument was equipped with ultraviolet $(280 \mathrm{~nm})$ and refractive index (RI) detectors, a PSS GRAM (polyester copolymer network) precolumn, and two PSS GRAM separation columns (particle size $=10 \mu \mathrm{m}$; pore size $=100$ and 10,000 $\AA)$ connected in series. The columns were operated at $60{ }^{\circ} \mathrm{C}$ and eluted with dimethyl sulfoxide (DMSO) with a $0.5 \%(\mathrm{w} / \mathrm{w}) \mathrm{LiBr}$ (lithium bromide) solution at a flow rate $0.5 \mathrm{~mL} \mathrm{~min}^{-1}$. Prior to injection, the powdery samples were completely dissolved in a DMSO/LiBr solution $\left(5 \mathrm{mg} \mathrm{mL}^{-1}\right)$ and filtered through a syringe filter (pore size: $0.45 \mu \mathrm{m}$ ). Pullulan standards in the molecular weight range of 342-708,000 Da were used for standard calibration. Weightaverage molecular weight $\left(M_{w}\right)$, number-average molecular weight $\left(M_{n}\right)$, and $Ð\left(M_{w} / M_{n}\right)$ were determined by WinGPC UniChrom software.

Proton and Phosphorus Nuclear Magnetic Resonance Spectroscopy $\left({ }^{1} H\right.$ and ${ }^{31} P$ NMR). The qualitative ${ }^{1} \mathrm{H}$ and quantitative ${ }^{31} \mathrm{P}$ NMR spectra were acquired on a Bruker Avance III HD $400 \mathrm{MHz}$ instrument with a BBFO probe equipped with a Z-gradient coil. Data processing was accomplished with MestReNova software V9.0.0, using a $90^{\circ}$ shifted square sine-bell apodization window. The phase and baseline corrections were applied. Measurements were performed at room temperature on at least three different samples.

Allyl functionalities were identified by ${ }^{1} \mathrm{H}$ NMR. Lignin samples $(25 \pm 0.1 \mathrm{mg})$ were dissolved in $550 \mu \mathrm{L}$ DMSO- $d_{6}$. The solvent signal at $2.50 \mathrm{ppm}$ was used as an internal reference signal for chemical shifts $(\delta)$. The spectra were recorded with a relaxation delay of $5 \mathrm{~s}$, a number of scans of 128 , and an acquisition time of $4.9 \mathrm{~s}$.

The quantification of the different hydroxyl groups in lignin was done via ${ }^{31} \mathrm{P}$ NMR. The sample preparation requires the phosphitylation of hydroxyl functionalities in the presence of an internal standard. ${ }^{30}$ 2-Chloro-4,4,5,5-tetramethyl-1,3-2-dioxaphospholane (TMDP, 95\%) was used as a phosphitylation reagent. The internal standard stock solution was prepared by dissolving $\approx 30 \mathrm{mg}$ of $\mathrm{N}$-hydroxy-5-norbornene-2,3-dicarboximide (NHND, 97\%) and $\approx 2.5$ $\mathrm{mg}$ of a relaxation reagent (chromium(III) acetylacetonate) in $500 \mu \mathrm{L}$ of anhydrous pyridine. Then, $25-35 \mathrm{mg}$ of lignin samples were dissolved in $200 \mu \mathrm{L}$ of an anhydrous pyridine/dimethylformamide $(1: 1 \mathrm{v} / \mathrm{v})$ mixture. After complete dissolution, $50 \mu \mathrm{L}$ of an internal standard stock solution and $150 \mu \mathrm{L}$ of TMDP were added. The formed precipitate was dissolved in $400 \mu \mathrm{L}$ of $\mathrm{CDCl}_{3}$ and left to rest for $20 \mathrm{~min}$ prior to analysis. The signal at $132.2 \mathrm{ppm}$ was used as an internal reference, arising from the phosphitylated product of TMDP with moisture. The data were acquired with an inverse gated decoupling pulse (zgig30) with an acquisition time of $1.67 \mathrm{~s}$, a relaxation delay of $10 \mathrm{~s}$, and a number of scans set to 128 .

Solid-State Cross-Polarization Magic Angle Spinning Carbon-13 Nuclear Magnetic Resonance (CP/MAS $\left.{ }^{13} \mathrm{C} N M R\right)$. CP/MAS ${ }^{13} \mathrm{C}$ NMR spectra were recorded with a Bruker Avance III AQS 400 SB instrument operating at $400 \mathrm{MHz}$. A double air-bearing two-channel probe head was used. Lignin samples (roughly $100 \mathrm{mg}$ ) were packed uniformly in a $4 \mathrm{~mm}$ zirconium oxide rotor. Measurements were performed at $296 \pm 1 \mathrm{~K}$. The MAS rate was $10 \mathrm{kHz}$. Acquisition was performed with a CP pulse sequence using a $2.95 \mu$ s proton $90^{\circ}$ pulse, an $800 \mu$ s ramped $(100 \%-50 \%)$ falling contact pulse, and a $2.5 \mathrm{~s}$ delay between repetitions. A SPINAL64 pulse sequence was used for ${ }^{1} \mathrm{H}$ decoupling. The Hartman-Hahn matching procedure was performed on glycine, and the chemical shift scale was calibrated to tetramethylsilane by assigning the data point of maximum intensity in $\alpha$-glycine carbonyl signal a chemical shift of $176.03 \mathrm{ppm}^{31}$

Thermogravimetric Analysis (TGA). The thermal stability of lignin samples was studied by TGA using a Mettler Toledo TGA/DSC1 instrument. Lignin samples with a mass of $10.5 \pm 1 \mathrm{mg}$ were placed in a ceramic crucible where they were kept for $10 \mathrm{~min}$ at $30^{\circ} \mathrm{C}$. Specimens were then heated at a rate of $5{ }^{\circ} \mathrm{C} \mathrm{min}^{-1}$ in a nitrogen

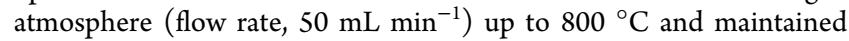
isothermally for $10 \mathrm{~min}$. Mettler-Toledo STARe software V15.00a was used to record all data. The measurements were performed in duplicate.

Differential Scanning Calorimetry (DSC). Thermal transitions were investigated by DSC using a Mettler Toledo DSC1 equipped with a sample robot. Measurements were performed with a heating/ cooling rate of $10^{\circ} \mathrm{C} \mathrm{min}{ }^{-1}$ in a nitrogen atmosphere (flow rate, 50

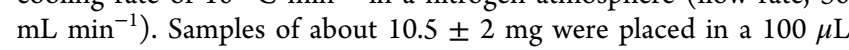


aluminum crucible and covered using a pierced lid. The DSC thermograms were recorded by heating the samples from room temperature up to $105{ }^{\circ} \mathrm{C}$ (first heating cycle) and isothermally kept for $20 \mathrm{~min}$ to erase any thermal history. They were then cooled to 20 ${ }^{\circ} \mathrm{C}$ and held isothermally for $10 \mathrm{~min}$. In the end, all samples were heated to $300{ }^{\circ} \mathrm{C}$ (second heating cycle). The onset point for the second heating cycle was taken as the glass transition temperature. The data were analyzed with Mettler-Toledo STARe software V15.00a. Each sample was measured in triplicate, and average values were reported.

Dynamic Mechanical Analysis (DMA). The viscoelastic properties of the lignin-based thermosets were obtained by a TA Instruments DMA Q800 equipped with a gas cooling accessory and tensile film clamps. The rectangular samples with approximate dimensions of 10.6 $\mathrm{mm} \times 5.2 \mathrm{~mm} \times 0.15 \mathrm{~mm}$ were subjected to a strain of $0.1 \%$ at $1 \mathrm{~Hz}$ with a preload force of $0.01 \mathrm{~N}$ (force track 125\%). Prior to testing, the samples were cooled and equilibrated at $-50^{\circ} \mathrm{C}$. Afterward, they were heated to a temperature of $250{ }^{\circ} \mathrm{C}$ at a rate of $3{ }^{\circ} \mathrm{C} \mathrm{min}-1$. Reported values of storage modulus $\left(E^{\prime}\right)$, loss modulus $\left(E^{\prime \prime}\right)$, and $\tan \delta$ (ratio between the dissipated energy to the stored energy) represent an average of three to six measurements. The $T_{g}$ was determined from the maximum of the $\tan \delta$ signal.

Wide-Angle and Small-Angle $X$-ray Scattering (WAXS and $S A X S)$. Scattering techniques were applied in order to investigate the samples' molecular and nanoscale structures and morphology. WAXS/SAXS experiments were conducted at the beamline P03, PETRA III, DESY (Hamburg, Germany). The used incident X-ray energy was $E=h \nu=11.7 \mathrm{keV}$. The sample-to-detector distance for WAXS was $\mathrm{SDD}_{\mathrm{WAXS}}=114.5 \pm 0.2 \mathrm{~mm}$ and for SAXS $\mathrm{SDD}_{\mathrm{SAXS}}=$ $4412 \pm 1 \mathrm{~mm}$ with a beam size of $\mathrm{H} \times \mathrm{V}, 16 \times 25 \mu \mathrm{m}^{2} .22$ Energy and SDD were calibrated using collagen calibrants for SAXS and lanthanum hexaboride for the WAXS setup. The SAXS scattering patterns were recorded using a Pilatus 300k $(172 \mu \mathrm{m} \times 172 \mu \mathrm{m}$, Dectris Ltd., Switzerland), and the WAXS patterns were recorded using a LAMBDA $4.5 \mathrm{M}(55 \mu \mathrm{m} \times 55 \mu \mathrm{m}, \mathrm{X}$-Spectrum $\mathrm{GmbH}$, Germany). The powdery lignin samples and cross-linkers were loaded in quartz capillaries $(2 \mathrm{~mm} \times 60 \mathrm{~mm} \times 0.01 \mathrm{~mm}$, diameter $\times$ length $\times$ wall thickness, Hilgenberg GmbH, Germany), mounted vertically, and scanned laterally. The lignin-based thermoset samples were mounted in a transmission holder and laterally scanned over $2 \mathrm{~mm}$ in order to check their homogeneity and prevent possible beam-induced changes. The exposure time was $100 \mathrm{~ms}$ for each position, and 20 images were summed for better statistics. Background measurements of the capillaries and of the empty transmission holder were subtracted from their corresponding samples. Radial integrations of the scattering patterns were performed using DPDAK V1.2.0. ${ }^{33}$ The extracted intensities were then analyzed using a Guinier-Porod model using SasView V5.0.0 software (www.sasview.org). In detail, the generalized Guinier-Porod model introduced by Hammouda for nonspherical scattering objects was used

$$
I(q)=\left\{\begin{array}{c}
\frac{G}{q^{s}} \exp \left(\frac{-q^{2} R_{g}^{2}}{3-s}\right) ; q \leq q_{1} \\
\frac{D}{q^{p}} ; q \geq q_{1}
\end{array}\right.
$$

where $I(q)$ is the scattered intensity. ${ }^{34} G$ and $D$ are the scaling parameters for the Guinier and Porod regime, respectively. $R_{g}$ is the Guinier radius, $s$ the dimensionality factor, and $p$ the Porod exponent. The dimensionality factor is defined as $s=0$ for spherical, $s=1$ for lamellae/platelets, and $s=2$ for rod-like objects. ${ }^{34}$

Scanning Electron Microscopy (SEM). The surface morphology of specimens was observed on a field emission Hitachi SEM S-4800 microscope. The images were captured at different magnifications (from 1000 to 4000 ) with an accelerating voltage of $1 \mathrm{kV}$ at a working distance of 3.7-3.9 $\mathrm{mm}$. The lignin powdery samples were placed on conductive carbon tape. All samples were $\mathrm{Pt} / \mathrm{Pd}$ sputtered for $20 \mathrm{~s}$ $(\approx 1.1 \mathrm{~nm}$ coating layer, measured with a quartz crystal microbalance) at a current of $80 \mathrm{~mA}$ with a Cressington 208HR sputter coater.
Tensile Testing. The tensile properties were measured by an Instron 5944 instrument (USA), equipped with a $500 \mathrm{~N}$ load cell. Rectangular samples with approximate dimensions of $30 \mathrm{~mm} \times 5.1$ $\mathrm{mm} \times 0.04 \mathrm{~mm}$ were prepared. The tensile grip distance was $20 \mathrm{~mm}$. The test was carried out in an environment-controlled room with temperature of $22 \pm 1{ }^{\circ} \mathrm{C}$ and relative humidity of $50 \% \pm 2 \%$. The crosshead speed was set to $5 \mathrm{~mm} / \mathrm{min}$.

\section{RESULTS AND DISCUSSION}

The present study focuses on how the properties of ligninbased thermosets can be tailored using cross-linkers with different degrees of functionalities. The effects of the fractionation process and functionalization on the morphology were studied to further support the structural evolution.

Purification, Fractionation, and Characterization of Raw Material. In order to investigate the efficiency of the washing process, analyses of ash and inorganics content were performed. As-received lignin ash content was lowered from $0.54 \% \pm 0.2 \%$ (in agreement with the value provided by Stora Enso, $0.58 \%)$ to $0.15 \% \pm 0.2 \%$ in washed lignin (KL Washed). The concentration of analyzed inorganics was diminished after the washing procedure, especially for $\mathrm{Na}, \mathrm{K}$, and $\mathrm{Cu}$, as shown in Table S1. It was observed that the total amount of inorganics was decreased by half. The washing process allows one to obtain lignin with higher purity and lower ash content.

LignoBoost Kraft lignin was sequentially solvent-fractionated to obtain more homogeneous fractions in terms of molecular weight and polydispersity. A schematic representation of the solvent fractionation is shown in Scheme S1. The yields and SEC results of the lignin fractions are found in Table S2 and Figure $S 1$. The $M_{w}$ and $Ð$ are relatively low for all soluble fractions compared to the initial Kraft lignin. An increasing trend in $M_{w}$ is seen from $\mathrm{KL} \_$EtOAc to KL_Acetone, while $Ð$ is decreasing gradually. ${ }^{9}$ The abundance of structural moieties, such as hydroxyl functionalities, is very important for further modifications. The aliphatic, phenolic, and carboxylic groups' contents were quantified by ${ }^{31} \mathrm{P}$ NMR (Figure S2), and the typical integration regions are represented in Table S3..$^{30,35,36}$ The concentration of aliphatic $\mathrm{OH}$ increases with increasing $M_{w}$, while the total amount of phenolic and carboxylic acid $\mathrm{OH}$ decreases. It is worth mentioning that the stability of the internal standard plays an important role in the precise $\mathrm{OH}$ groups measurements done via ${ }^{31} \mathrm{P}$ NMR (for these measurements, the number of scans was set to 256). ${ }^{37}$ The measurements of $\mathrm{OH}$ groups present in the $\mathrm{KL}$ EtOH fraction $4 \mathrm{~h}$ after preparation showed an increase in functionalities by $\approx 5 \%-10 \%$. Prolonged time between preparation of samples and measurements $(10 \mathrm{~h})$ led to an $\approx 10 \%-20 \%$ increase in total $\mathrm{OH}$ groups as a result of possible internal standard degradation. $^{38}$

The thermal behavior of the initial lignin and recovered fractions was investigated by TGA and DSC (Figure S3). Lignin degradation occurs over a wide range of temperatures, with a major mass loss between 200 and $650{ }^{\circ} \mathrm{C}$. Degradation above $100{ }^{\circ} \mathrm{C}$ is mainly attributed to the devolatilization of moisture and residue solvents. ${ }^{39}$ The fragmentation of phenyl propane side chains occurs between $\approx 100$ and $\approx 300{ }^{\circ} \mathrm{C}$. In the next stage, the monomeric phenols were released, and almost $50 \%$ of the initial weight was lost. ${ }^{40}$ The thermal stability of lignin soluble fractions increases with increasing $M_{w}$. The soluble fraction with the highest $M_{w}$ and highest concentration of condensed phenolic $\mathrm{OH}$ generates the most residue at 800 

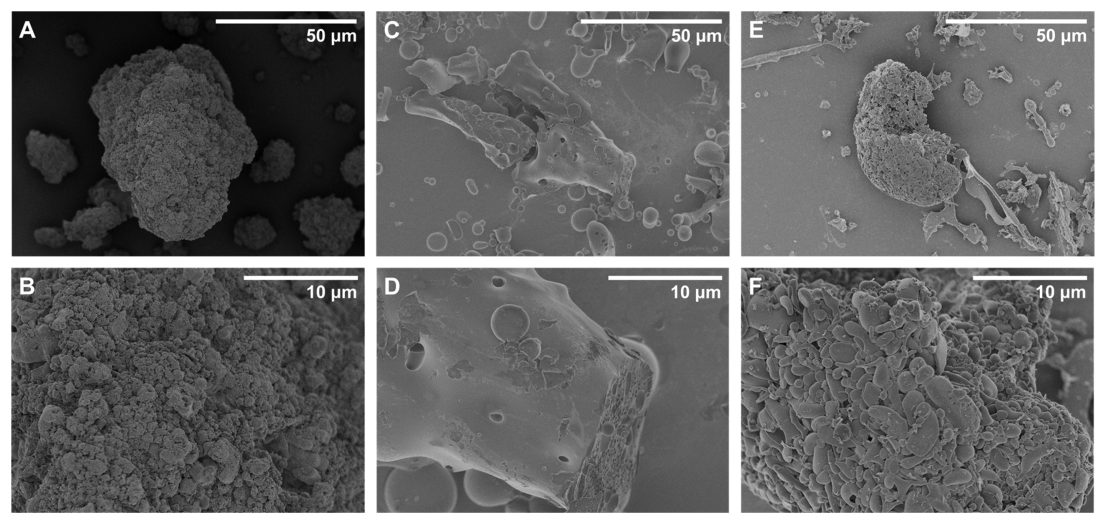

Figure 1. Representative SEM images of the initial lignin (KL_Initial: A, B), the ethanol soluble fraction (KL_EtOH: C, D), and the allylated lignin (A-KL_EtOH: E, F) at lower magnification (top row) and at higher magnification (bottom row).

${ }^{\circ} \mathrm{C}$. The thermal transition appearing in DSC thermograms of lignin fractions can be associated with the increase of $T_{g}$ as a function of $M_{w}$ due to the lower mobility of longer chains. The effects of the hydrogen bond interactions between various hydroxyl functionalities and high aromaticity explain the relatively high glass transition temperature. ${ }^{41}$ More detailed characterizations of lignin fractions were reported in earlier works. ${ }^{18,42-44}$ The final morphology and physical structure of the initial Kraft lignin and the retrieved ethanol fraction after freeze-drying were assessed with SEM analysis and X-ray scattering to allow for a comparison with the allylated resin and the final thermosets.

These results show that it is important to have control of the entire process in retrieving the starting materials for the resins synthesis. It also shows that a wide variety of starting materials can be derived from crude lignin by appropriate adaption of the refining process.

Selective Allylation of Ethanol Soluble Fraction. The $\mathrm{KL} \mathrm{EtOH}$ fraction was chosen for further modification. This fraction was the most promising one for selective allylation because of its high yield from the fractionation procedure, high solubility in reaction media, and low enough $M_{w}$ to ensure good processeability. ${ }^{21,22}$ The allylation process was studied by ${ }^{1} \mathrm{H},{ }^{31} \mathrm{P}$, and $\mathrm{CP} / \mathrm{MAS}{ }^{13} \mathrm{C}$ NMR. The ${ }^{1} \mathrm{H}$ NMR spectra (Figure S4) before and after allylation show a signal at 3.77 ppm, which was assigned to the methoxy group. Three new signals appeared between 6.19 and 5.59 ppm, 5.52 and 4.80 $\mathrm{ppm}$, and 4.71 and $4.06 \mathrm{ppm}$, which were assigned to the allyl ether group. ${ }^{16}$ From the $\mathrm{CP} / \mathrm{MAS}{ }^{13} \mathrm{C}$ NMR spectra (Figure S5), it can be seen that after chemical modification with allyl chloride, new signals appeared at 69.8, 73.3, 117.2, and 133.9 ppm as a result of allylic group incorporation. The signal at $55.3 \mathrm{ppm}$ was assigned to the aromatic methoxy group. During chemical modification, the phenolic hydroxyl groups were allylated, and the signal assigned to the phenolic $\mathrm{C}-\mathrm{OH}$ carbons at $146.8 \mathrm{ppm}$ decreased. ${ }^{45}{ }^{31} \mathrm{P}$ NMR spectra (Figure S6) shows that up to $85 \%$ of phenolic $\mathrm{OH}$ was converted into allyl aryl ethers, but the amount of aliphatic and carboxylic $\mathrm{OH}$ remained unchanged. SEC measurements of four A-KL_EtOH from different batches showed no variability of $M_{w}$ within the detection limit of the technique. $M_{w}$ increased slightly from 1900 to $2100 \mathrm{~g} / \mathrm{mol}$ as a result of allyl functional groups incorporation (Figure S7).

The allylation process was studied by FT-IR (Figure S8). The intensity of the hydroxyl groups stretching vibration band at $3400 \mathrm{~cm}^{-1}$ decreased as a consequence of phenolic hydroxyls modification. Due to introduction of the allyl group, the absorption bands at 2934 and $2845 \mathrm{~cm}^{-1}$ increased for $\mathrm{C}-\mathrm{H}$ stretching vibrations of $\mathrm{CH}$ and $\mathrm{CH}_{2}$ groups. The observed four new signals are considered as evidence of successful chemical modification. The stretching vibration bands of $=\mathrm{CH}$ and $\mathrm{C}=\mathrm{C}$ were observed at 3077 and 1647 $\mathrm{cm}^{-1}$. The two bands corresponding to the deformation vibration of a double bond $\left(-\mathrm{CH}=\mathrm{CH}_{2}\right)$ appeared at 990 and $925 \mathrm{~cm}^{-1} \cdot{ }^{16,17}$ The typical aromatic $\mathrm{C}=\mathrm{C}$ stretching vibration absorption bands at 1585 and $1510 \mathrm{~cm}^{-1}$ were present in both the unmodified fraction and allylated lignin. The methoxy groups present in $\mathrm{KL} \_$EtOH remained unchanged after chemical modification and showed two absorption bands at 1455 and $1420 \mathrm{~cm}^{-1}$. 46

The morphological characteristics of KL_Initial, KL_EtOH and A-KL_EtOH were studied by $\mathrm{SEM}_{\overline{1}}$, and images are illustrated in Figure 1 and Figure S9. Initial powdery lignin particles exhibit near spherical geometry with a size of a few micrometers to hundreds of micrometers, with a high surface roughness (Figure 1A, B). The freeze-drying of the KL_EtOH fraction (at concentration of $\approx 19 \mathrm{mg} / \mathrm{mL}$ ) leads to a sheet-like morphology with a smooth surface and a porous interior due to aggregation of lignin particles in the presence of ice crystals (Figure 1C, D). ${ }^{47,48}$ The pores are irregularly distributed and have a spherical shape. The porosity depends on the lignin concentration. By decreasing the concentration of dispersed lignin to $\approx 7 \mathrm{mg} / \mathrm{mL}$, the porosity increases (Figure S10). On the other hand, by increasing the concentration of dispersed lignin to $\approx 40 \mathrm{mg} / \mathrm{mL}$, the porosity decreases (Figure S11). ${ }^{49}$ The allylation of the lignin drastically changes the morphology of the sheet-like geometry (Figure 1E, F). A-KL_EtOH has the larger specific surface area and appears like an aggregation of small grains. The reason for these differences in lignin morphology between KL_EtOH and A-KL_EtOH can be related to the different hydrophilicity and surface activity of lignin chains. ${ }^{50}$

In order to further characterize the initial lignin, ethanol soluble fraction, and allylated lignin, transmission SAXS measurements were performed. The 1D scattering intensities were extracted from the 2D SAXS pattern and fitted using the Guinier-Porod model (eq 1) to gain information on the size, dimensionality, and surface roughness of the nanostructure features inside the material. The information obtained at the nanoscale can be imposed directly to macroscale properties such as mechanical stiffness. ${ }^{4}$ The $1 \mathrm{D}$ scattering intensities $I(q)$, with $q$ being the scattering vector 


$$
\vec{q}=q=\frac{4 \pi}{\lambda} \sin 2 \theta
$$

along with their corresponding fits are shown in Figure S12. The full $q$-scale can be fitted with good precision through a single Guinier-Porod model. Hence, it can be assumed that only one type of feature is present in the material. The fitted parameters $\left(R_{g}, s\right.$, and $\left.p\right)$ can be found in Figure S13. The dimensionality parameter, $s$, is represented in Figure 2 and

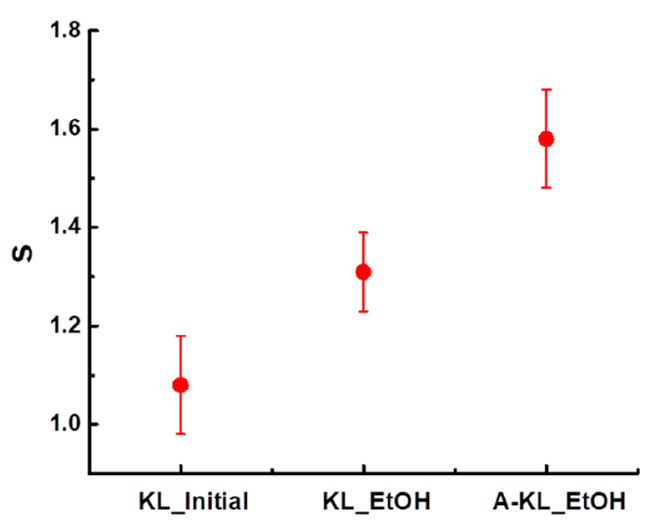

Figure 2. Dimensionality parameter, $s$, of the SAXS fitting results using the Guinier-Porod model assumption for KL_Initial, $\mathrm{KL} \_\mathrm{EtOH}$, and A-KL_EtOH.

increases from $1.08 \pm 0.1$ for $\mathrm{KL}$ Initial to $1.31 \pm 0.08$ for $\mathrm{KL} \_$EtOH and finally to $1.58 \pm \overline{0} .1$ for A-KL_EtOH. This stems from a change in morphology from a platelet-like structure to a more rod-shaped one while simultaneously slightly decreasing the Guinier radius after allylation. Hammouda showed that a Porod exponent $p=3$ describes particles with a very rough surface, whereas $p=4$ stands for smooth surfaces. ${ }^{34}$ The Porod exponent remains the same for $\mathrm{KL} \_\mathrm{EtOH}$ and $\mathrm{A}-\mathrm{KL} \_\mathrm{EtOH}(\approx 3.73)$ and decreases slightly for $\bar{K} L$ Initial $(\approx 3.56)$, indicating that the surface roughness on the nanoscale features is comparable.

Curing Performance of Allylated Lignin Resins Using Different Thiol Cross-linkers. The three-dimensional networks were generated by thermal curing of lignin resins with three different thiol cross-linkers (Scheme 2). The network formation was investigated by FT-IR, and the spectra before and after curing for all resins are shown in Figure S14.
Moreover, the zoomed-in region corresponding to the absorption bands of thiol and ene functionalities are shown in Figure 3. In order to further enhance chain mobility, a curing temperature of $125{ }^{\circ} \mathrm{C}$ was chosen so that it was above the $T_{g}$ of the allylated lignin $\left(\approx 100{ }^{\circ} \mathrm{C}\right)$.

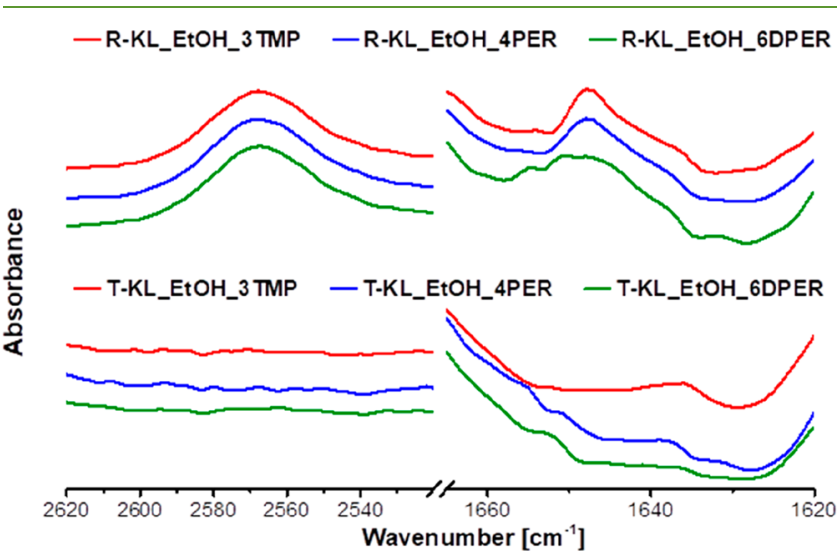

Figure 3. Enlarged regions of the FT-IR spectra before and after curing showing the thiol $\left(2568 \mathrm{~cm}^{-1}\right)$ and ene $\left(1647 \mathrm{~cm}^{-1}\right)$ consumption. The spectra were shifted for better visualization.

During the stoichiometric reaction, the stretching vibration of the $\mathrm{S}-\mathrm{H}$ group at $2568 \mathrm{~cm}^{-1}$ disappeared after curing, which signifies the successful incorporation of the thiol groups in the polymer network. The band at $1647 \mathrm{~cm}^{-1}$ corresponding to stretching vibrations of the $\mathrm{C}=\mathrm{C}$ group disappeared almost entirely. The level of residual unsaturation is within the experimental error limits of the FT-IR analysis technique. The reproducibility of the developed thermoset preparation makes this a desirable approach for future applications.

Physical and Mechanical Properties of Lignin-Based Thermosets. An ideal case for network formation is when each functional group of a certain cross-linker molecule interacts with a double bond from different lignin chains to form a sulfur-carbon bond. ${ }^{51}$ This situation is less common when heterogeneous compounds, such as lignin, are involved. The topological defects as dangling chains, entanglements, and primary loops (when at least two thiol groups of the same molecule interact with ene groups of the same lignin chain and the remaining thiol groups interact with ene functionalities of others lignin chains) can give rise to regions with different

\section{Scheme 2. Simple Illustration of the Curing Process}

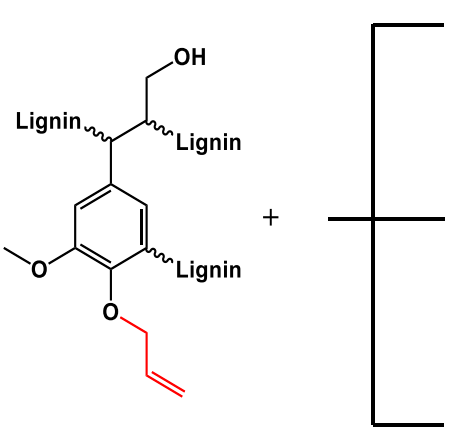

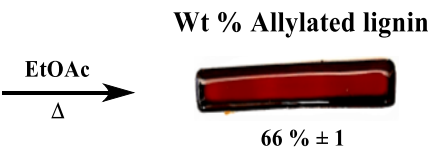
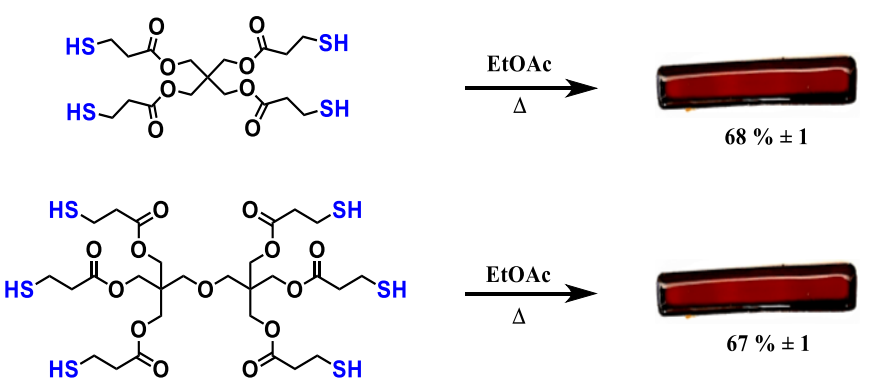

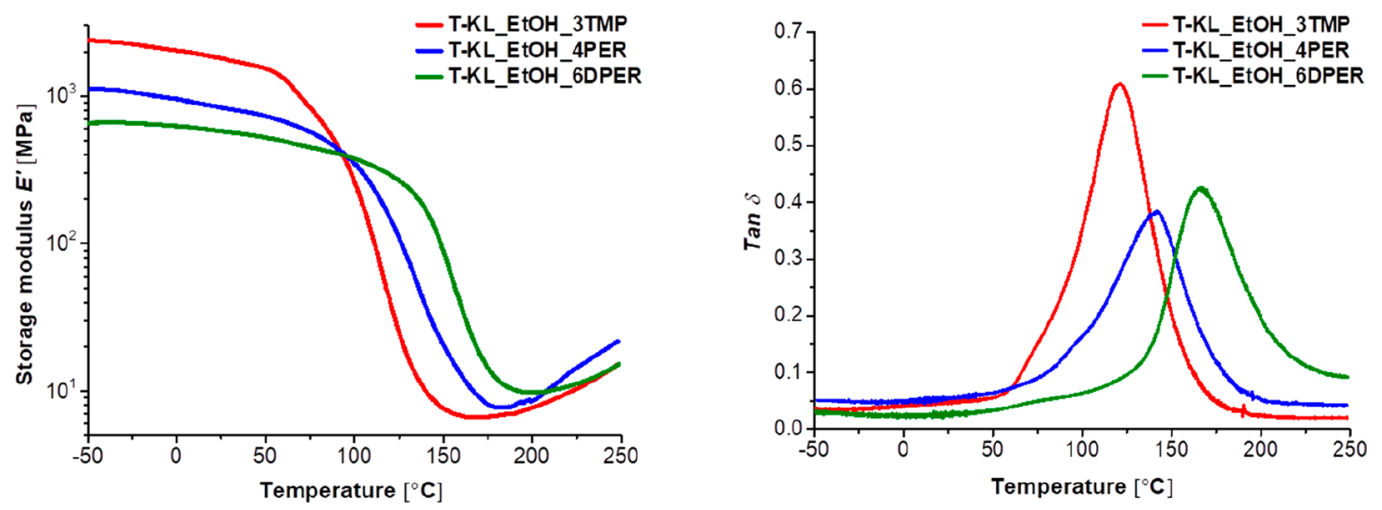

Figure 4. Representative DMA curves of the different thermosets. Storage modulus $\left(E^{\prime}\right)$ illustrated on the left and $\tan \delta$ illustrated on the right.

Table 2. Storage Modulus and $\tan \delta$ of Various Lignin-Based Thermosets

\begin{tabular}{|c|c|c|c|}
\hline & T-KL_EtOH_3TMP & T-KL_EtOH_4PER & T-KL_EtOH_6DPER \\
\hline$T_{g \tan \delta}\left({ }^{\circ} \mathrm{C}\right)$ & $120 \pm 9$ & $149 \pm 5$ & $157 \pm 8$ \\
\hline$E^{\prime}{ }_{-50}{ }^{\circ} \mathrm{C}(\mathrm{MPa})$ & $1750 \pm 750$ & $990 \pm 150$ & $750 \pm 110$ \\
\hline
\end{tabular}

mobility affecting the mechanical properties of cured resins. ${ }^{51}$ Therefore, mechanical properties of the resulting thermosets were investigated by DMA. The storage modulus and $\tan \delta$ signals are shown in Figure 4 and the loss modulus in Figure S15. Jawerth et al. revealed that the mechanical properties of lignin-based thermosets can be tuned by using different lignin fractions and a single cross-linker agent. ${ }^{22}$ In the present study, it has instead been demonstrated that the mechanical properties can be tailored by varying the cross-linker and maintaining the lignin component. The storage modulus and $\tan \delta$ values of different three-dimensional networks are affected mainly by the rigid aromatic lignin backbone and the cross-link density. The thermosets' storage modulus shows a stable behavior at temperatures below $T_{g}$ with relatively high values ranging from around 750 to $2200 \mathrm{MPa}$. When the temperature was increased, the storage modulus dropped significantly and reached a minimum in a temperature range between 150 and $200{ }^{\circ} \mathrm{C}$. The drop is associated with the thermosets' softening effect which manifests itself in the glass transition region. The difference between the minimum storage modulus values of the thermosets is the result of the differences in cross-link density, which increases with the number of cross-link functionalities. Meanwhile, the unexpected differences in storage modulus below $T_{g}$ is more difficult to explain. The $E^{\prime}{ }_{-50}^{\circ} \mathrm{C}$ values are reported in Table 2 . It is apparent that the storage modulus exhibits an inverse trend compared to cross-link density below $T_{g}$. The exact reason for this is currently under investigation. One possible explanation can be that the amount of primary loops increases with increasing the number of cross-link functionalities. ${ }^{52}$ The primary loops consisting of thio-ether bonds are flexible and can give rise to softer regions within the thermoset network. It is well established that the thiol-ether linkage is significantly more flexible compared to a $\mathrm{C}-\mathrm{C}$ bond. The results indicate that the distribution of the thiol-ether cross-links differ between the different thermosets. The stiffness of lignin-based networks is most likely reduced by the plasticizer effect of the loops and the cross-link distribution within the network. This can partially explain the reverse effect of cross-link density on the storage modulus at temperatures below $T_{g}$.
The maxima of the $\tan \delta$ signals was attributed to the glass transition temperature. As a result of incorporating rigid aromatic lignin structures, the $T_{g}$ occurs at temperatures above $100{ }^{\circ} \mathrm{C}$, which is relatively high for a thiol-ene material. ${ }^{26} T_{g}$ increases with the rise of cross-link functionalities as a result of higher cross-link density. The $T_{g}$ of cured systems also depends strongly on the van der Waals dispersion interactions between polymeric chains, hydrogen bonds between unmodified $\mathrm{OH}$ groups, and $\pi-\pi$ interactions of the aromatic rings. The shape of the $\tan \delta$ signal reflects the homogeneity of the network. The sharper and narrower the signal is, the more homogeneous the thermoset is. All obtained films show a relatively narrow signal. A significant shift toward higher temperatures was observed in the maxima of $\tan \delta$ from T-KL_EtOH_3TMP to T-KL_EtOH_6DPER (Table 2). By increasing the cross-linkdensity, the $\bar{T}_{g}$ increases as a result of restrictions of chain mobility. The increase in $T_{g}$ is significant showing that both the lignin structure (as previously reported ${ }^{22}$ ) as well as how the lignin resin is combined with different cross-linkers can be used to tailor the final thermoset properties. A $T_{g}$ above $100{ }^{\circ} \mathrm{C}$ makes these thermosets suitable for a wide range of applications, for example, adhesives and composite matrices.

The molecular and nanoscale morphological features present within the cured resins were investigated by SAXS and WAXS. The 1D scattering intensities, $I(q)$, along with their corresponding model fits for specific cross-linkers and associated lignin-based thermosets, are shown in Figures S16 and S17. SAXS fitting results from the Guinier-Porod model assumption of the $s, p$, and $R_{g}$ parameters for employed crosslinkers and thermosets are shown in Figure S13. The dimensionality factor, $s$, for all thermosets has a small deviation $(1.05 \pm 0.03)$ and transforms from a more rod-like geometry (as shown before for A-KL_EtOH) into a platelet one. The repeating structures are rather smooth, with a $p$ value of approximately $3.79 .{ }^{34} R_{g}$ indicates the size of the observed structure domains within the thermosets. It can be seen that after incorporating the cross-linker molecules the repeating features' size increases by $\approx 1.3 \mathrm{~nm}$ for all three thermosets.

The lignin morphology at the molecular level was studied by WAXS. The inter- and intramolecular interactions between the aromatic rings highly affect the lignin aggregation. ${ }^{53}$ The 
Table 3. $\pi-\pi$ Stacking Interactions between Repeating Features ${ }^{a}$

$\pi-\pi($ sandwich $)(\AA)$
Content (\%)
$\pi-\pi(\mathrm{T}$-shaped) $(\AA)$
Content (\%)

T-KL_EtOH_3TMP
$4.41 \pm 0.03$
$87 \pm 1$
$7.48 \pm 0.07$
$12 \pm 1$

T-KL_EtOH_4PER
$4.40 \pm 0.03$
$87 \pm 1$
$7.48 \pm 0.06$
$12 \pm 1$

T-KL_EtOH_6DPER

$4.39 \pm 0.03$

$89 \pm 1$

$7.39 \pm 0.04$

$11 \pm 1$

${ }^{a}$ Distances were calculated from the fitting of the peaks at the WAXS measurements. ${ }^{56}$.

WAXS intensities clearly show previously reported peaks which occur due to $\pi-\pi$ stacking of the aryl ether structures within lignin (Figure S18). ${ }^{22,54,53}$ These structures have been fitted individually with Gaussian functions (Figure S19) to determine their content, and all values are presented in Table 3. The aromatic lignin chains can interact with each other by $\pi-\pi$ interactions in two different modes: sandwich and T-shaped $\pi-\pi$ arrangements. ${ }^{53}$ The sandwich stacking is the favored geometry and constitutes approximately up to $88 \%$ $\pm 1 \%$ of all thermosets. The ratio between the two $\pi-\pi$ stacking remained unchanged by varying the cross-linking agent. This further supports the hypothesis that it is the thiolether bond distribution within the thermosets that determines the stiffness below $T_{g}$. Predominant sandwich stacking can also contribute to the understanding of the native lignin structure. $\beta$-O-4 linkages are more likely to form than $\beta-5$ in the presence of sandwich $\pi-\pi$ arrangements. ${ }^{54}$ Because of lignin heterogeneity, crystalline domains were not observed. Morphology of all thermosets can be considered to be highly comparable even though different cross-linkers were used.

The thermal stability of lignin-cured resins was assessed by TGA, and the thermograms are shown in Figure S20. By removing the majority of phenolic $\mathrm{OH}$ and by achieving high cross-link density, the thermal stability of the obtained materials was improved. The three different thermosets show good stability up to $250{ }^{\circ} \mathrm{C}$, and there are no big differences between them.

A preliminary uniaxial tensile testing was conducted on sample T-KL_EtOH_3TPM, and the representative stressstrain curve is shown in Figure S21. The results showed a Young's modulus, $E$, of $4.8 \pm 1.5 \mathrm{GPa}$, and a tensile stress at break, $\sigma_{b}$, of $68 \pm 6 \mathrm{MPa}$. These values are well in line with commonly used thermoset polymers for composite applications. ${ }^{57}$ This implies that the present thermosets have a potential in applications such as composites as thermoset matrices.

\section{CONCLUSIONS}

In this work, it was shown that starting with a heterogeneous renewable feedstock such as lignin it was possible to produce thermosets with controlled and tunable properties by varying the cross-linking agent. The sequentially solvent fractionation approach leads to the production of more homogeneous lignin fractions with different chemical properties. The retrieved ethanol soluble fraction was chosen for further selective allylation toward phenolic hydroxyl groups. Morphological aspects after fractionation and chemical modifications were investigated at different levels. At the microlevel, the sheet-like morphology of ethanol soluble fractions after allylation changed into aggregates of small grains. The morphological features at nanoscale studied via SAXS showed a change from a platelet-like structure for $\mathrm{KL}$ EtOH to a more rod-shaped one for A-KL_EtOH. Meanwhile, $R_{g}$ decreased slightly from 5.06 $\mathrm{nm}$ for $\mathrm{K} \overline{\mathrm{L}}_{-} \mathrm{EtOH}$ to $4.85 \mathrm{~nm}$ for A-KL_EtOH. The allylated lignin was thermally cured through a thiol-ene reaction. Three multifunctional thiol cross-linkers were used to tune the mechanical and morphological properties of the resin.

The obtained lignin-based thermosets displayed a relatively high $T_{g}$ for a thiol-ene material. With increasing the number of functionalities of the cross-linking agent, the $T_{g}$ of the thermosets also increases as a result of higher cross-link density. The large amount of the aromatic rings present in lignin and their $\pi-\pi$ stacking contributes significantly to the high $T_{g}$ of the thermosets. The WAXS measurements demonstrated that two different types of $\pi-\pi$ interactions are present within the thermosets (sandwich and T-shaped) and that the ratio between them stayed constant for all thermosets. SAXS studies revealed that the $R_{g}$ of repeating features within the thermosets increased by $\approx 1.3 \mathrm{~nm}$ as a result of thiol cross-linking agent incorporation. SAXS and WAXS studies indicated that all thermosets followed similar morphological evolution.

A significant improvement in thermal stability was noticed after the curing reaction: all samples were stable up to $250{ }^{\circ} \mathrm{C}$ and can be used for specific applications that require high $T_{g}$ and thermal stability.

This work highlights how the morphological and mechanical characteristics of the produced lignin-based thermosets influence the structure-property relationship. The obtained results can help gain better understanding of lignin behavior during fractionation, chemical modification, and network formation processes.

\section{ASSOCIATED CONTENT}

\section{Supporting Information}

The Supporting Information is available free of charge at https://pubs.acs.org/doi/10.1021/acssuschemeng.0c07580.

ICP-OES results for initial and washed lignin; fractionation procedure and SEC results for initial lignin, retrieved fractions, and allylated product; identification and quantification of different $\mathrm{OH}$ groups by ${ }^{1} \mathrm{H},{ }^{31} \mathrm{P}$, and $\mathrm{CP} / \mathrm{MAS}{ }^{13} \mathrm{C} \mathrm{NMR}$ and the spectra of ethanol soluble fraction and allylated lignin; TGA and DSC thermograms of initial lignin and retrieved fractions; FT-IR spectra of ethanol soluble fraction, allylated lignin, and the three different resins before and after curing; SEM images of initial lignin, ethanol soluble fraction, and allylated lignin; SAXS and WAXS 1D plots with corresponding fits and results from the GuinierPorod model assumption; TGA thermograms and loss modulus curves for the three different thermosets; representative stress-strain curve for the T-KL_EtOH_3TMP sample (PDF) 


\section{AUTHOR INFORMATION}

\section{Corresponding Author}

Mats Johansson - Department of Fiber and Polymer Technology, Wallenberg Wood Science Center (WWSC) and Department of Fiber and Polymer Technology, Division of Coating Technology, KTH Royal Institute of Technology, SE10044 Stockholm, Sweden; (1) orcid.org/0000-0003-32015138; Email: matskg@kth.se

\section{Authors}

Iuliana Ribca - Department of Fiber and Polymer Technology, Wallenberg Wood Science Center (WWSC) and Department of Fiber and Polymer Technology, Division of Coating Technology, KTH Royal Institute of Technology, SE-10044 Stockholm, Sweden; (1) orcid.org/0000-0002-8127-9183

Marcus E. Jawerth - Department of Fiber and Polymer Technology, Wallenberg Wood Science Center (WWSC) and Department of Fiber and Polymer Technology, Division of Coating Technology, KTH Royal Institute of Technology, SE10044 Stockholm, Sweden; (1) orcid.org/0000-0003-42268593

Calvin J. Brett - Department of Fiber and Polymer Technology, Wallenberg Wood Science Center (WWSC), Department of Fiber and Polymer Technology, Division of Coating Technology, and Department of Engineering Mechanics, KTH Royal Institute of Technology, SE-10044 Stockholm, Sweden; Deutsches Elektronen-Synchrotron (DESY), 22607 Hamburg, Germany; 이잉.org/00000001-5789-6299

Martin Lawoko - Department of Fiber and Polymer Technology, Wallenberg Wood Science Center (WWSC), KTH Royal Institute of Technology, SE-10044 Stockholm, Sweden; 이이.org/0000-0002-8614-6291

Matthias Schwartzkopf - Deutsches Elektronen-Synchrotron (DESY), 22607 Hamburg, Germany; ○ orcid.org/00000002-2115-9286

Andrei Chumakov - Deutsches Elektronen-Synchrotron (DESY), 22607 Hamburg, Germany; 10 orcid.org/00000003-3195-9356

Stephan V. Roth - Department of Fiber and Polymer Technology, Division of Coating Technology, KTH Royal Institute of Technology, SE-10044 Stockholm, Sweden; Deutsches Elektronen-Synchrotron (DESY), 22607 Hamburg, Germany; 10 orcid.org/0000-0002-6940-6012

Complete contact information is available at: https://pubs.acs.org/10.1021/acssuschemeng.0c07580

\section{Author Contributions}

The manuscript was written through contribution of all authors. All authors have given approval to the final version of the manuscript.

\section{Notes}

The authors declare no competing financial interest.

\section{ACKNOWLEDGMENTS}

The research has been funded by the Knut and Alice Wallenberg Foundation through the Wallenberg Wood Science Center at KTH Royal Institute of Technology. The authors acknowledge DESY (Hamburg, Germany), a member of the Helmholtz Association HGF, for the provision of experimental facilities. Parts of this research were carried out at P03 beamline at PETRA III. C.J.B. and S.V.R. acknowledge financial support from the DESY strategic fund (DSF) under the grant "Investigation of processes for spraying and spraycoating of hybrid cellulose-based nanostructures". The authors would like to thank X-Spectrum $\mathrm{GmbH}$ for the support in installing the LAMBDA $4.5 \mathrm{M}$ and Dr. André Rothkirch (DESY) for IT support. The authors acknowledge the Treesearch Research Infrastructure and Jasna Stevanic and Tomas Larsson from RISE for the support with the CP/MAS ${ }^{13} \mathrm{C}$ NMR analysis. We thank Prof. Dan Zenkert (KTH Royal Institute of Technology) for helpful discussions on the tensile test measurements and Yi Yang (KTH Royal Institute of Technology) for the ICP-OES measurements. We would also like to thank Anastasia Riazanova and Céline Montanari (KTH Royal Institute of Technology) for their valuable suggestions during the SEM measurements.

\section{REFERENCES}

(1) Auvergne, R.; Caillol, S.; David, G.; Boutevin, B.; Pascault, J. P. Biobased Thermosetting Epoxy: Present and Future. Chem. Rev. 2014, 114 (2), 1082-1115.

(2) vom Saal, F. S.; Hughes, C. An Extensive New Literature Concerning Low-Dose Effects of Bisphenol A Shows the Need for a New Risk Assessment. Environ. Health Perspect. 2005, 113 (8), 926933.

(3) Vanholme, R.; Demedts, B.; Morreel, K.; Ralph, J.; Boerjan, W. Lignin Biosynthesis and Structure. Plant Physiol. 2010, 153 (3), 895905.

(4) Gandini, A. Polymers from Renewable Resources: A Challenge for the Future of Macromolecular Materials. Macromolecules 2008, 41 (24), 9491-9504.

(5) Cui, C. Z.; Sun, R. K.; Argyropoulos, D. S. Fractional Precipitation of Softwood Kraft Lignin: Isolation of Narrow Fractions Common to a Variety of Lignins. ACS Sustainable Chem. Eng. 2014, 2 (4), 959-968.

(6) Sadeghifar, H.; Wells, T.; Le, R. K.; Sadeghifar, F.; Yuan, J. S.; Ragauskas, A. J. Fractionation of Organosolv Lignin Using Acetone:Water and Properties of the Obtained Fractions. ACS Sustainable Chem. Eng. 2017, 5 (1), 580-587.

(7) Crestini, C.; Lange, H.; Sette, M.; Argyropoulos, D. S. On the structure of softwood kraft lignin. Green Chem. 2017, 19 (17), 41044121.

(8) Brodin, I.; Sjoholm, E.; Gellerstedt, G. Kraft lignin as feedstock for chemical products: The effects of membrane filtration. Holzforschung 2009, 63 (3), 290-297.

(9) Duval, A.; Vilaplana, F.; Crestini, C.; Lawoko, M. Solvent screening for the fractionation of industrial kraft lignin. Holzforschung 2016, 70 (1), 11-20.

(10) García, A.; Toledano, A.; Serrano, L.; Egüés, I.; González, M.; Marín, F.; Labidi, J. Characterization of lignins obtained by selective precipitation. Sep. Purif. Technol. 2009, 68 (2), 193-198.

(11) Cederholm, L.; Xu, Y. S.; Tagami, A.; Sevastyanova, O.; Odelius, K.; Hakkarainen, M. Microwave processing of lignin in green solvents: A high-yield process to narrow-dispersity oligomers. Ind. Crops Prod. 2020, 145, 112152.

(12) Sadeghifar, H.; Ragauskas, A. Perspective on Technical Lignin Fractionation. ACS Sustainable Chem. Eng. 2020, 8 (22), 8086-8101.

(13) Fache, M.; Darroman, E.; Besse, V.; Auvergne, R.; Caillol, S.; Boutevin, B. Vanillin, a promising biobased building-block for monomer synthesis. Green Chem. 2014, 16 (4), 1987-1998.

(14) Wong, Z. J.; Chen, K. F.; Li, J. Formation of Vanillin and syringaldehyde in an Oxygen Delignification Process. Bioresources 2010, 5 (3), 1509-1516.

(15) Fache, M.; Boutevin, B.; Caillol, S. Vanillin, a key-intermediate of biobased polymers. Eur. Polym. J. 2015, 68, 488-502.

(16) Over, L. C.; Meier, M. A. R. Sustainable Allylation of Organosolv Lignin with Diallyl Carbonate and detailed Structural 
Characterization of modified Lignin. Green Chem. 2016, 18 (1), 197207.

(17) Zoia, L.; Salanti, A.; Frigerio, P.; Orlandi, M. Exploring Allylation and Claisen Rearrangement as a Novel Chemical Modification of Lignin. BioResources 2014, 9, 6540-6561.

(18) Gioia, C.; Lo Re, G.; Lawoko, M.; Berglund, L. Tunable Thermosetting Epoxies Based on Fractionated and Well-Characterized Lignins. J. Am. Chem. Soc. 2018, 140 (11), 4054-4061.

(19) Sadeghifar, H.; Cui, C. Z.; Argyropoulos, D. S. Toward Thermoplastic Lignin Polymers. Part 1. Selective Masking of Phenolic Hydroxyl Groups in Kraft Lignins via Methylation and Oxypropylation Chemistries. Ind. Eng. Chem. Res. 2012, 51, 1671316720.

(20) Buono, P.; Duval, A.; Verge, P.; Averous, L.; Habibi, Y. New Insights on the Chemical Modification of Lignin: Acetylation versus Silylation. ACS Sustainable Chem. Eng. 2016, 4 (10), 5212-5222.

(21) Jawerth, M.; Johansson, M.; Lundmark, S.; Gioia, C.; Lawoko, M. Renewable Thiol-Ene Thermosets Based on Refined and Selectively Allylated Industrial Lignin. ACS Sustainable Chem. Eng. 2017, 5 (11), 10918-10925.

(22) Jawerth, M. E.; Brett, C. J.; Terrier, C.; Larsson, P. T.; Lawoko, M.; Roth, S. V.; Lundmark, S.; Johansson, M. Mechanical and Morphological Properties of Lignin-Based Thermosets. ACS Appl. Polym. Mater. 2020, 2, 668-676.

(23) Hoyle, C. E.; Bowman, C. N. Thiol-Ene Click Chemistry. Angew. Chem., Int. Ed. 2010, 49 (9), 1540-1573.

(24) Nair, D. P.; Cramer, N. B.; McBride, M. K.; Gaipa, J. C.; Shandas, R.; Bowman, C. N. Enhanced Two-Stage Reactive Polymer Network Forming Systems. Polymer 2012, 53 (12), 2429-2434.

(25) Guzmán, D.; Ramis, X.; Fernández-Francos, X.; Serra, A. Preparation of click thiol-ene/thiol-epoxy thermosets by controlled photo/thermal dual curing sequence. RSC Adv. 2015, 5 (123), 101623-101633.

(26) Hoyle, C. E.; Lee, T. Y.; Roper, T. Thiol-Enes: Chemistry of the Past with Promise for the Future. J. Polym. Sci., Part A: Polym. Chem. 2004, 42 (21), 5301-5338.

(27) Kade, M. J.; Burke, D. J.; Hawker, C. J. The Power of Thiol-ene Chemistry. J. Polym. Sci., Part A: Polym. Chem. 2010, 48 (4), 743750 .

(28) Braun, J. v.; Murjahn, R. Haftfestigkeit organischer Reste (IV). Ber. Dtsch. Chem. Ges. B 1926, 59 (6), 1202-1209.

(29) Claudino, M.; Mathevet, J.-M.; Jonsson, M.; Johansson, M. Bringingd-limonene to the scene of bio-based thermoset coatings via free-radical thiol-ene chemistry: macromonomer synthesis, UVcuring and thermo-mechanical characterization. Polym. Chem. 2014, 5 (9), 3245-3260.

(30) Meng, X.; Crestini, C.; Ben, H.; Hao, N.; Pu, Y.; Ragauskas, A. J.; Argyropoulos, D. S. Determination of hydroxyl groups in biorefinery resources via quantitative 31P NMR spectroscopy. Nat. Protoc. 2019, 14 (9), 2627-2647.

(31) Larsson, P. T.; Wickholm, K.; Iversen, T. A CP/MAS 13C NMR investigation of molecular ordering in celluloses. Carbohydr. Res. 1997, 302, 19-25.

(32) Buffet, A.; Rothkirch, A.; Döhrmann, R.; Körstgens, V.; Abul Kashem, M. M.; Perlich, J.; Herzog, G.; Schwartzkopf, M.; Gehrke, R.; Müller-Buschbaum, P.; Roth, S. V. P03, the microfocus and nanofocus $\mathrm{X}$-ray scattering (MiNaXS) beamline of the PETRA III storage ring: the microfocus endstation. J. Synchrotron Radiat. 2012, 19 (4), 647653.

(33) Benecke, G.; Wagermaier, W.; Li, C.; Schwartzkopf, M.; Flucke, G.; Hoerth, R.; Zizak, I.; Burghammer, M.; Metwalli, E.; MüllerBuschbaum, P.; Trebbin, M.; Förster, S.; Paris, O.; Roth, S. V.; Fratzl, P. A customizable software for fast reduction and analysis of large $\mathrm{X}$ ray scattering data sets: applications of the new DPDAK package to small-angle X-ray scattering and grazing-incidence small-angle X-ray scattering. J. Appl. Crystallogr. 2014, 47 (5), 1797-1803.

(34) Hammouda, B. A new Guinier-Porod model. J. Appl. Crystallogr. 2010, 43 (4), 716-719.
(35) Argyropoulos, D. Quantitative Phosphorus-31 NMR Analysis of Six Soluble Lignins. J. Wood Chem. Technol. 1994, 14 (1), 65-82.

(36) Granata, A.; Argyropoulos, D. S. 2-Chloro-4,4,5,5-tetramethyl1,32-dioxaphospholane, a Reagent for the Accurate Determination of the Uncondensed and Condensed Phenolic Moieties in Lignins. J. Agric. Food Chem. 1995, 43 (6), 1538-1544.

(37) Korntner, P.; Sumerskii, I.; Bacher, M.; Rosenau, T.; Potthast, A. Characterization of technical lignins by NMR spectroscopy: optimization of functional group analysis by 31P NMR spectroscopy. Holzforschung 2015, 69 (6), 807-814.

(38) Stücker, A.; Podschun, J.; Saake, B.; Lehnen, R. A novel quantitative 31P NMR spectroscopic analysis of hydroxyl groups in lignosulfonic acids. Anal. Methods 2018, 10 (28), 3481-3488.

(39) Zhao, X. B.; Liu, D. H. Chemical and thermal characteristics of lignins isolated from Siam weed stem by acetic acid and formic acid delignification. Ind. Crops Prod. 2010, 32 (3), 284-291.

(40) Fenner, R. A.; Lephardt, J. O. Examination of the Thermal Decomposition of Kraft Pine Lignin by Fourier Transform Infrared Evolved Gas Analysis. J. Agric. Food Chem. 1981, 29 (4), 846-849.

(41) Park, S. Y.; Kim, J. Y.; Youn, H. J.; Choi, J. W. Fractionation of lignin macromolecules by sequential organic solvents systems and their characterization for further valuable applications. Int. J. Biol. Macromol. 2018, 106, 793-802.

(42) Tagami, A.; Gioia, C.; Lauberts, M.; Budnyak, T.; Moriana, R.; Lindstrom, M. E.; Sevastyanova, O. Solvent fractionation of softwood and hardwood kraft lignins for more efficient uses: Compositional, structural, thermal, antioxidant and adsorption properties. Ind. Crops Prod. 2019, 129, 123-134.

(43) Olsén, P.; Jawerth, M.; Lawoko, M.; Johansson, M.; Berglund, L. A. Transforming technical lignins to structurally defined starcopolymers under ambient conditions. Green Chem. 2019, 21 (9), $2478-2486$.

(44) Giummarella, N.; Lindén, P. A.; Areskogh, D.; Lawoko, M. Fractional Profiling of Kraft Lignin Structure: Unravelling Insights on Lignin Reaction Mechanisms. ACS Sustainable Chem. Eng. 2020, 8 (2), 1112-1120.

(45) Hatfield, G. R.; Maciel, G. E.; Erbatur, O.; Erbatur, G. Qualitative and Quantitative Analysis of Solid Lignin Samples by Carbon-13 Nuclear Magnetic Resonance Spectrometry. Anal. Chem. 1987, 59 (1), 172-179.

(46) Over, L. C.; Hergert, M.; Meier, M. A. R. Metathesis Curing of Allylated Lignin and Different Plant Oils for the Preparation of Thermosetting Polymer Films with Tunable Mechanical Properties. Macromol. Chem. Phys. 2017, 218 (16), 1700177.

(47) Liu, W. S.; Yao, Y. M.; Fu, O. L.; Jiang, S. H.; Fang, Y. C.; Wei, Y.; Lu, X. H. Lignin-derived carbon nanosheets for high-capacitance supercapacitors. RSC Adv. 2017, 7 (77), 48537-48543.

(48) Chen, F. G.; Shi, W. B.; Zhou, D.; Zhang, Q. Q.; Lv, L. P.; Sun, J. T.; Yang, L. P. Nanostructured thin lignin-derived carbon sheets as excellent reinforcement fillers in polypropylene. RSC Adv. 2018, 8 (65), 37472-37479.

(49) Chen, F. G.; Liu, W. S.; Shahabadi, S. I. S.; Xu, J. W.; Lu, X. H. Sheet-Like Lignin Particles as Multifunctional Fillers in Polypropylene. ACS Sustainable Chem. Eng. 2016, 4 (9), 4997-5004.

(50) Rojas, O.; Salager, J. L. Surface activity of bagasse lignin derivatives found in the spent liquor of soda plants. Tappi J. 1994, 77 (3), 169-174.

(51) Li, C. L.; Johansson, M.; Sablong, R. J.; Koning, C. E. High performance thiol-ene thermosets based on fully bio-based poly(limonene carbonate)s. Eur. Polym. J. 2017, 96, 337-349.

(52) Bowman, C. N.; Kloxin, C. J. Toward an Enhanced Understanding and Implementation of Photopolymerization Reactions. AIChE J. 2008, 54 (11), 2775-2795.

(53) Deng, Y.; Feng, X.; Zhou, M.; Qian, Y.; Yu, H.; Qiu, X. Investigation of Aggregation and Assembly of Alkali Lignin Using Iodine as a Probe. Biomacromolecules 2011, 12 (4), 1116-1125.

(54) Terashima, N.; Yoshida, M.; Hafren, J.; Fukushima, K.; Westermark, U. Proposed supramolecular structure of lignin in 
softwood tracheid compound middle lamella regions. Holzforschung 2012, 66 (8), 907-915.

(55) Hatakeyama, T.; Hatakeyama, H. Temperature-dependence of X-ray diffractograms of amorphous lignins and polystyrenes. Polymer 1982, 23 (3), 475-477.

(56) Sinnokrot, M. O.; Valeev, E. F.; Sherrill, C. D. Estimates of the $\mathrm{Ab}$ Initio Limit for pi-pi Interactions: The Benzene Dimer. J. Am. Chem. Soc. 2002, 124 (36), 10887-10893.

(57) Åström, B. T. Manufacturing of Polymer Composites, 1st ed.; Champan \& Hall: London, 1997; p 143. 